\title{
Clinical and magnetic resonance imaging correlation in acute spinal cord injury
}

\author{
S Ramón ${ }^{1}$, R Domínguez ${ }^{2}$, L Ramírez ${ }^{1}$, M Paraira $^{2}$, M Olona ${ }^{3}$, T Castelló ${ }^{1}$ and L García Fernández ${ }^{1}$ \\ ${ }^{1}$ Spinal Injury Unit; Rehabilitation Service, ${ }^{2}$ Radiology Service, ${ }^{3}$ Department of Preventive Medicine, Traumatology \\ and Rehabilitation University Hospital Vall d'Hebrón, Vall d'Hebrón Hospitals, Po Vall d'Hebrón 119-129, 08035 \\ Barcelona, Spain
}

\begin{abstract}
The aim of this study was to correlate traumatic spinal cord injury (SCI) patients' outcome with magnetic resonance imaging (MRI) performed within the first 15 days following trauma. We retrospectively analyzed 55 SCI patients. Upon admission, 28 were diagnosed as having a complete SCI $(51 \%)$, versus 27 with an incomplete SCI (49\%). All of the patients with a normal pattern on MRI (four cases), had an incomplete SCI, whereas all patients (15 cases) presenting with a hemorrhage pattern (Type 1$)$ had a complete SCI $(P=0.0001)$. Fourteen of the 15 individuals $(93.4 \%)$ with the edema pattern (Type II) had an incomplete SCI $(P=0.001)$, while the other patient had neurological deterioration, and a syrinx was noted 2 years later $(6.6 \%)$. Among the 10 individuals showing a contusion pattern (Type III), seven were admitted with an incomplete SCI $(70 \%)$ and three with a complete SCI $(30 \%)$. The compression pattern tends to be associated with a complete SCI in $77.8 \%$ (seven of nine patients). All patients with a transection pattern on MRI (two cases) were clinically diagnosed as having a complete SCI. Early functional prognosis may be established on the basis of clinical presentation of SCI and associated MRI. Cord hemorrhage and transection are irreversible, while edema has a potential for neurological recovery. Cord contusion tends to be associated with an incomplete SCI, unlike the compression pattern, in which the prognosis depends on the degree of the initial neurological damage.
\end{abstract}

Keywords: spinal cord injury; spine fracture; magnetic resonance imaging; prognostic factors

\section{Introduction}

The evaluation of emergency patients with clinical signs of spinal cord injury (SCI) should initially include assessment of the neurological injury and radiographs of the spine. Although computed tomography provides more details of bone injury, magnetic resonance imaging (MRI) has become the technique of choiceparticularly if no fracture is seen-in order to assess spinal cord damage and to exclude soft tissue injuries such as an extradural hematoma or a disc herniation.

The diagnosis of the initial clinical injury is critical in order to predict an accurate functional prognosis, a challenge for clinicians and radiologists. Classically, neurological recovery according to the Frankel classification $^{1}$ has been the only predictive value in SCI. $^{2-4}$ Motor score was used to predict recovery in other studies. $^{4-6}$ Kulkarni $^{7}$ described three patterns of MRI in SCI patients (hemorrhage, edema and contusion) correlating them with the neurological status at the time of discharge. Other authors added cord transection, ${ }^{8-10}$ and Silberstein ${ }^{10}$ has recently studied spinal cord compression on MRI.

Correspondence: Dr L García Fernández, Spinal Injury Unit Chief.
The aim of this study was to correlate the initial clinical SCI and the associated outcome with MRI performed during the early phase of SCI in order to establish clinical and imaging prognostic factors involved in neurological recovery.

\section{Methods}

A retrospective study was performed in 55 patients presenting with acute SCI due to trauma who were admitted to the Spinal Injury Unit of the Rehabilitation Department at the University Hospital Vall d'Hebron from January 1990 to December 1993 and who underwent MRI within 15 days following trauma.

The following data were analyzed: etiological data related to trauma and clinical data, such as neurological level, motor index score (MIS), SCI type using the ASIA (American Spinal Injury Association) Classification System. ${ }^{11,12}$ We have subdivided the incomplete spinal cord syndromes into anterior, central, Brown-Séquard, posterior, conus medullaris and 'non-classifiable' (anterior and posterior) syndromes. Cauda equina lesions were excluded from 
the study. Neurological recovery of the SCI was determined on the basis of the neurological level, motor and sensory scores and ASIA grade, at the time of discharge and in the final evaluation. Radiological data were classified according to the type of spinal fracture, with specification of the site.

MRI was performed on a MR unit 1.5 T Magneton SP or 1.0 T Magneton Impact (Siemens, MR System) using surface coils with $4 \mathrm{~mm}$ slice thickness and 220 or $280 \mathrm{~mm}$ fields of view. Sagittal T1-weighted images (WI) were obtained with a spin echo (SE) sequence $(\mathrm{TR} / \mathrm{TE}=580 / 15 \mathrm{msec} ; 3$ acquisition; matrix $=$ $192 \times 256)$. Sagittal T2-WI were made with a multiecho technique (SE TR/TE $=2200 / 20-80 \mathrm{msec}$; 1 acquisition; $192 \times 256$; flip angle $70^{\circ}$ ). Transaxial T1WI SE sequences were obtained $(\mathrm{TR} / \mathrm{TE}=710 / 15 ; 3$ acquisition; $192 \times 256$ ), and T2-WI were occasionally obtained by means of a gradient recalled acquisition sequences $\left(\mathrm{TR} / \mathrm{TE}=510 / 15 ; 250 \times 250\right.$; flip angle $\left.15^{\circ}\right)$. In the evaluation of SCI, we have broadened the patterns described by Kulkarni ${ }^{7}$ up to six patterns. The criteria used to define them are based on alterations in the signal intensity of the spine detected in the sequences on $\mathrm{T} 1$ and T2-WI with respect to the time elapsed since the trauma. These criteria are as follows: Normal pattern: No signal abnormalities of the cord in any sequence, despite neurological deficit. Type I pattern (hemorrhage) (Figure 1): within the first $72^{\circ} \mathrm{h}$ after the trauma, the spinal cord on T1-WI is heterogeneous; on T2-WI, a large central area with low signal intensity surrounded by a thin high intensity peripheral ring (extracellular methemoglobin). At $72 \mathrm{~h}$ to the first week from the trauma, the spinal cord shows hyperintensity on $\mathrm{T} 1$ and T2-WI. Type II pattern (Edema) (Figure 2): normal images on T1-WI with high signal intensity on T2-WI. Type III pattern (Contusion or mixed) (I and II) (Figure 3): Normal images on $\mathrm{T} 1$, while on $\mathrm{T} 2$ the spinal cord presents with a small central area of isointensity and a thick peripheral ring of high intensity, which persists through the subacute phase. Compression pattern (Figure 4): severe obliteration of the spinal cord, with significant alteration of its morphology preventing detection of signal alterations such as bleeding. Transection pattern (Figure 5): sagittal discontinuity of spinal cord. a

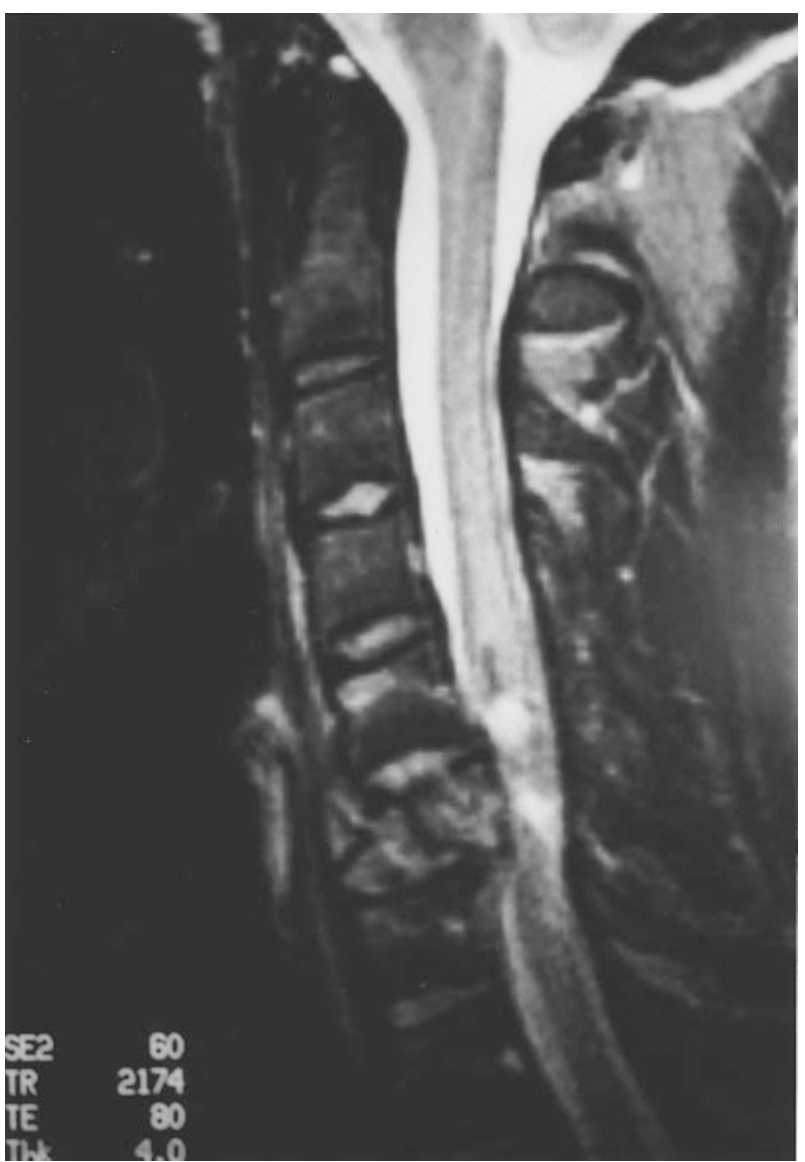

b

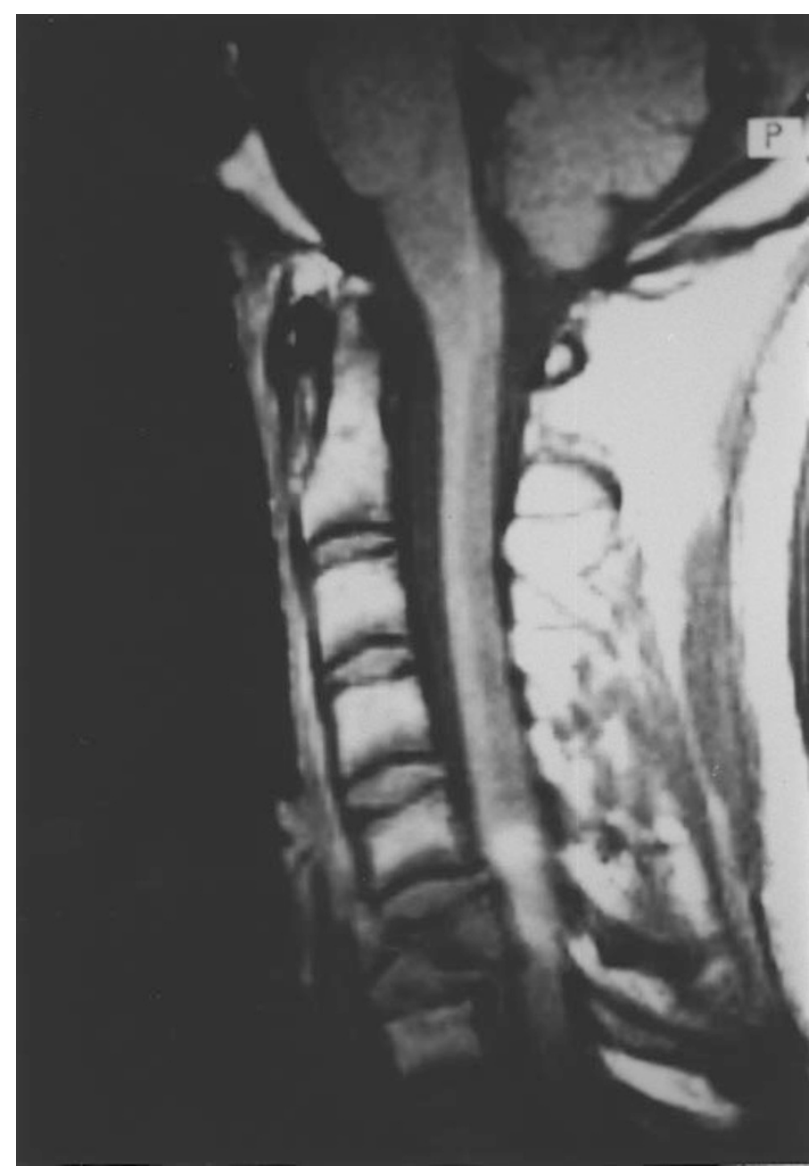

Figure 1 This 18-year-old man suffered a motorcycle accident. He presented with a complete C6 tetraplegia secondary to a C6 burst fracture. MR imaging obtained 14 days after the injury demonstrated on T2-WI a decreased intensity cord lesion at C5C6 level (a) and a hyperintensity signal on T1-WI (b), which suggested hemorrhage 


20

a

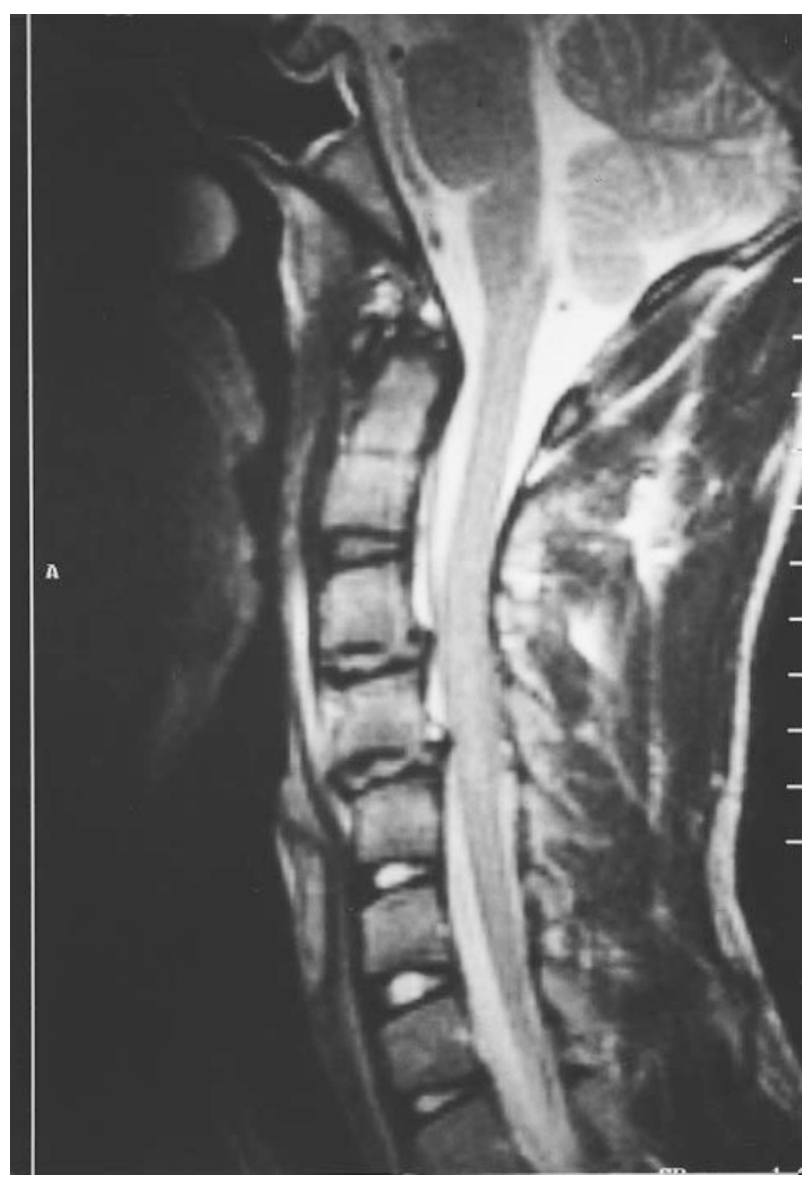

b

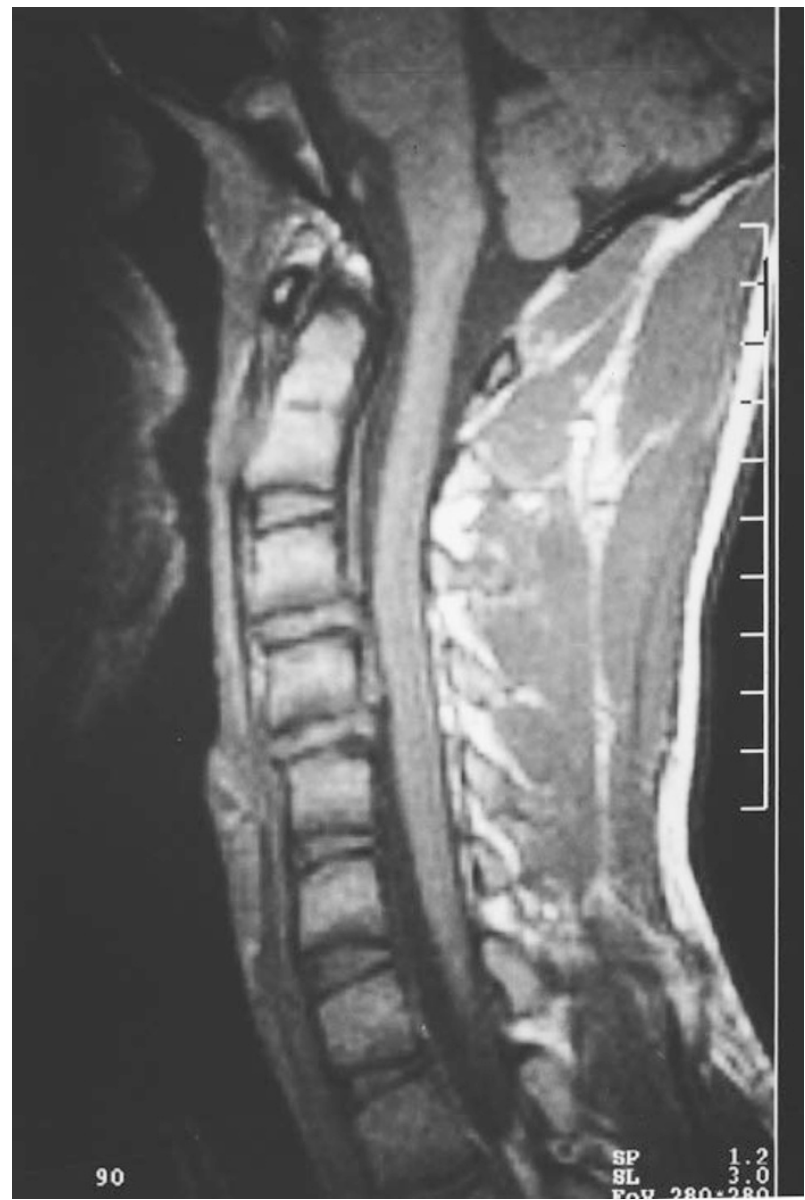

Figure 2 This is an 18-year-old man presented with a central cord syndrome after a hyperextension C3-C5 fracture-dislocation from a motor-vehicle accident. The T2-WI obtained three days after the trauma showed hyperintensity behind the C4 vertebral body. The cord was enlarged and disc herniations were visualized at C3-C4 and C4-C5 levels (a). T1-WI adjacent to the C4 level was isointense, indicating cord edema (b). Upon admission, patient's motor index score (MIS) was 92 and improved to 99 at discharge $(7.6 \%$ of recovery)

Therapeutic data included use of methylprednisolone therapy following the criteria established by Bracken $^{13}$ and the treatment of vertebral fracture, whether conservative or surgical.

\section{Statistical methods}

BMDP statistical programs were used. The Chi-squared test was used in order to study the association of two categorical variables. The association of a quantitative variable with a categorical variable was performed using the Kruskall-Wallis test. The Friedman test was used to analyze neurological outcome. The statistical significance level accepted was $P<=0.05$.

\section{Results}

Fifty-five patients with an acute SCI due to trauma were studied, 44 men $(80 \%)$ and 11 women $(20 \%)$ with a mean age of 32.4 years and standard deviation (SD) of 15 (range 14-76). The most frequent etiology of the trauma was motor vehicle accident in 32 patients $(58 \%)$, followed by accidental fall in 13 patients $(23.6 \%)$, work-related accident in six patients $(11 \%)$, sports-related accident in three patients $(5.4 \%)$ and a penetrating injury in one $(2 \%)$.

The traumatic lesion of the spine resulted in fracture-dislocation in 22 patients $(40 \%)$, burst fracture in 12 patients $(21.8 \%)$ and compression fracture in 11 patients $(20 \%)$. No fracture was observed in 10 patients $(18.2 \%)$. The fracture sites were as follows: cervical vertebrae in 10 patients $(22.3 \%)$, thoracic spine in $25(55.4 \%)$ and the thoracolumbar junction in $10(22.3 \%)$.

The initial neurological level in 18 patients was cervical $(32.3 \%)$, thoracic in $27(49.5 \%)$ and lower than or equal to T12 in $10(18.2 \%)$. According to the ASIA scale upon admission, 28 were complete, ASIA 
a

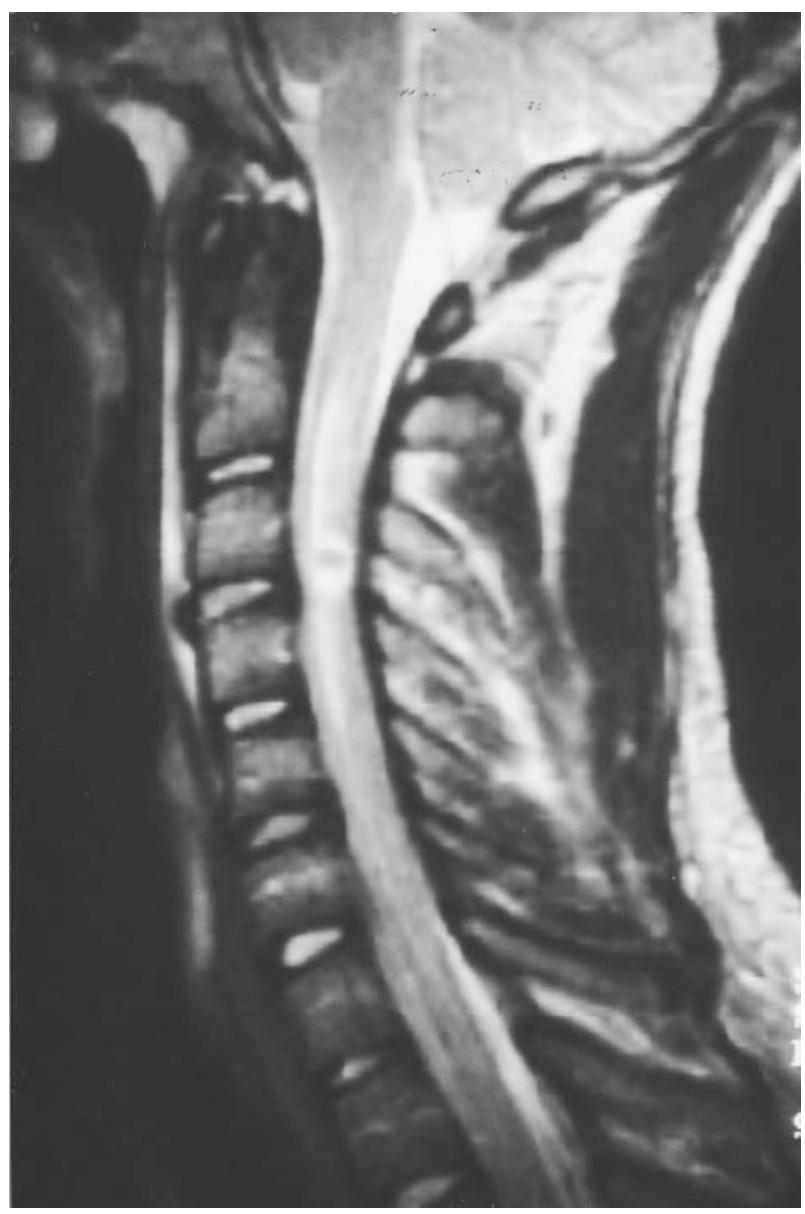

b

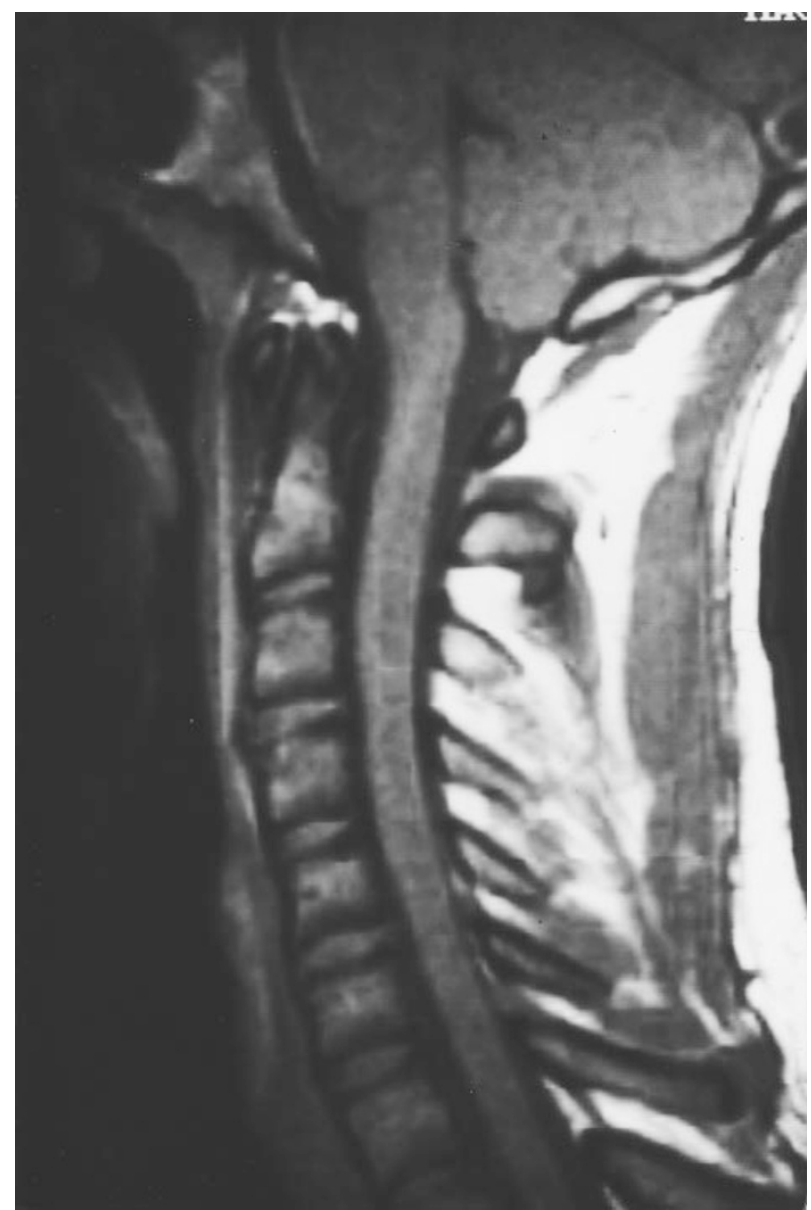

Figure 3 This 19-year-old man suffered a centro-lateral cord syndrome after an extension injury from a bicycle accident. The T2-WI three days following trauma showed a disruption of the anterior longitudinal ligament between $\mathrm{C} 3$ and $\mathrm{C} 4$ and a central isointensity surrounded by a peripheral rim of hyperintensity (a). T1-WI was normal (b), which suggested cord contusion. This patient had an initial motor score of 30 , and after 3 months it rose up to 79 ( $163 \%$ of recovery)

A $(51 \%)$, seven ASIA B $(12.75 \%), 13$ were ASIA C $(23.5 \%)$ and seven were ASIA D (12.75\%). The distribution of the 27 patients with incomplete SCI syndromes was as follows: seven anterior $(25.9 \%)$, three central $(11.1 \%)$, six Brown-Séquard $(22.3 \%)$, seven conus medullaris $(25.9 \%)$ and four 'nonclassifiable syndrome' $(14.8 \%)$.

On the basis of the MRI patterns, Normal Pattern was found in four patients $(7.3 \%)$, Type I Pattern (hemorrhage) in $15(27.3 \%)$, Type II Pattern (edema) $(27.3 \%)$ in 15 , Type III Pattern (contusion) in 10 (18.1\%), Compression Pattern in nine (16.4\%) and Transection Pattern in two (3.6\%). Other MRI findings are described in Table 1 . The distribution of MRI findings with respect to complete or incomplete SCI is shown in Figure 6.

The evolution of the motor MIS in each MRI pattern was studied in incomplete SCI, as well as quadriparetic and paraparetic patients (Figures 7 and 8). Clinical outcome of the SCI in relation to MRI obtained in the early period ( 1 to 15 days) was analyzed (Tables 2 and 3):

\section{Normal pattern}

The four patients with a normal MRI pattern had an incomplete SCI (ASIA C or D) on admission, which progressed towards $\mathrm{D}$ or $\mathrm{E}$. None of the patients showed any neurological sequelae (ASIA E) in the final evaluation. The mean MIS increase from 77 upon admission to 97.5 at discharge and 100 by the time of follow-up ( $30 \%$ of recovery).

\section{Type I pattern (hemorrhage)}

The 15 patients with this pattern were admitted with complete SCI, ASIA A, with 14 of them showing no change neither at discharge nor in follow-up. A 41 year-old man with complete C6 SCI ascended to C4 and died 1 month after admission from pulmonary 


\begin{tabular}{|c|c|}
\hline n & $\begin{array}{l}\text { SCI and MRI } \\
\text { S Ramón et al }\end{array}$ \\
\hline
\end{tabular}

a

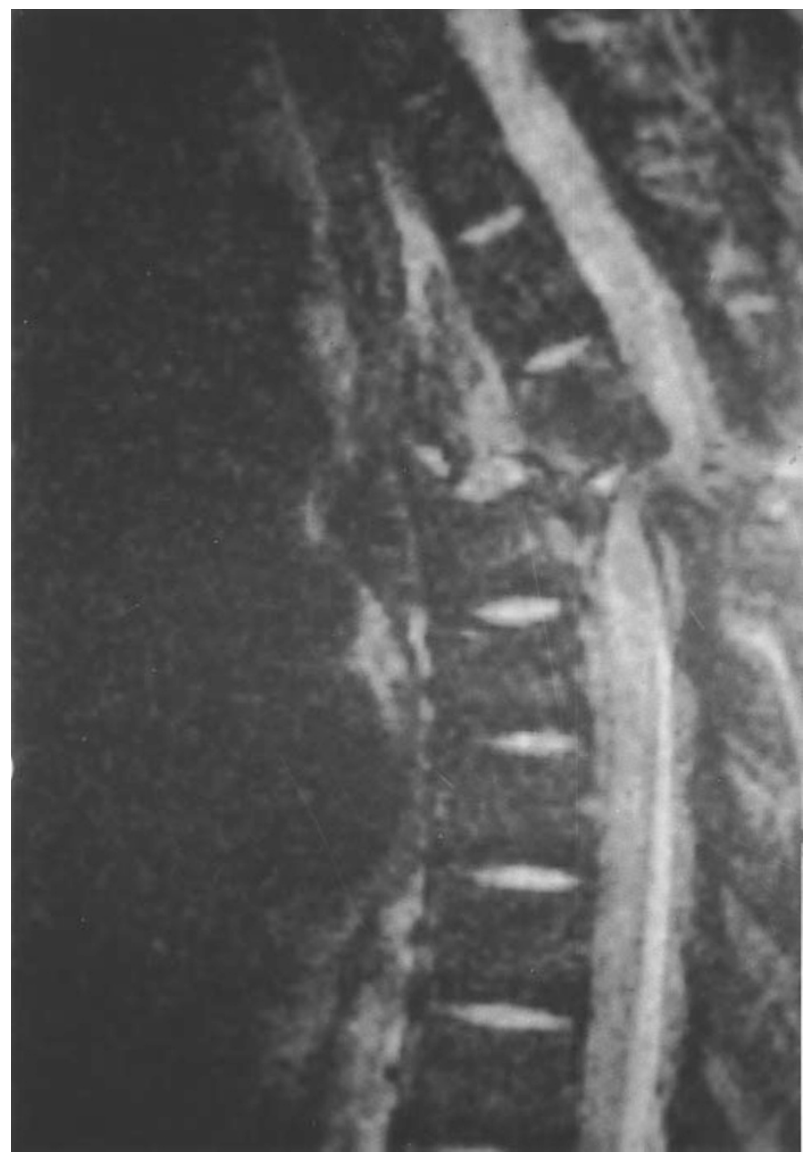

b

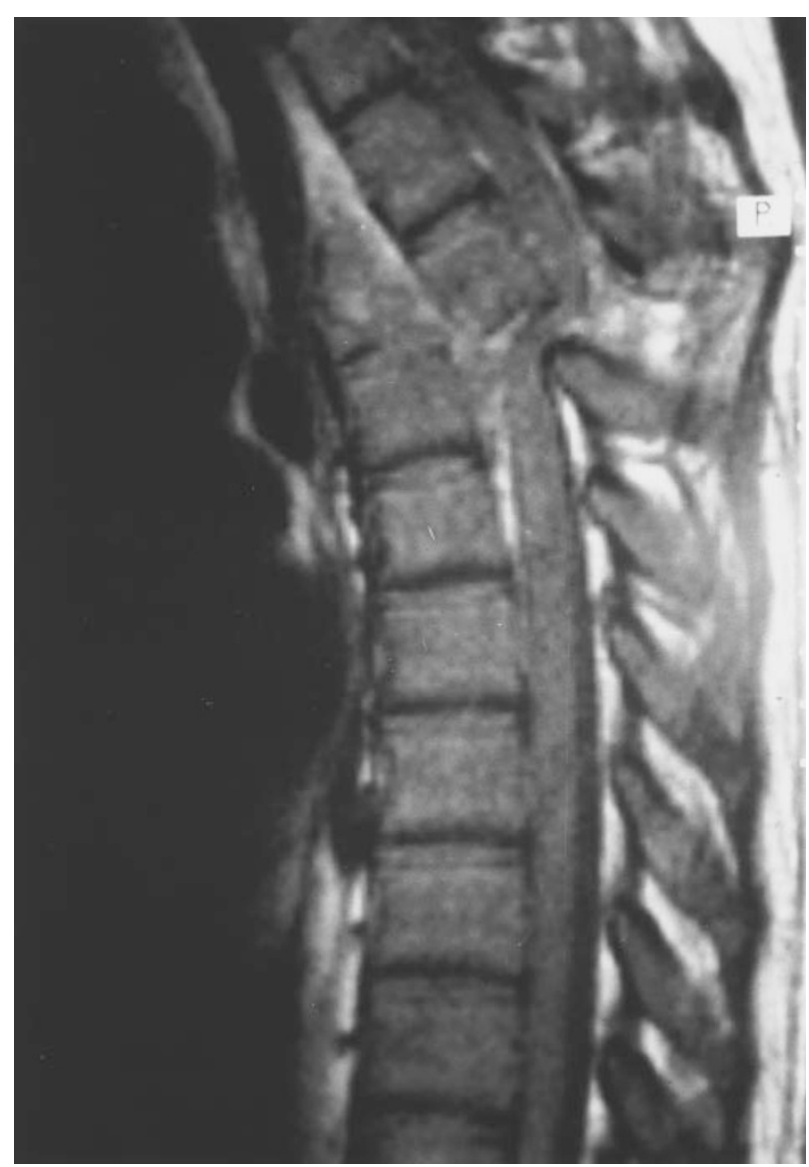

Figure 4 A 23-year-old man was involved in a motor-vehicle accident and suffered a complete T4 SCI secondary to a severe T3-T4 fracture-dislocation. MRI obtained 9 days after the accident demonstrated on T2 and T1-WI the retropulsed posterior wall of the T3 vertebrae ( $\mathrm{a}$ and $\mathrm{b}$ ). This decrease of the spinal canal and encroachment of spinal canal hindered visualization of the spinal cord, suggesting a compression pattern. Motor score on admission was 50 without any further change

thromboembolism. The mean MIS increased from 42 at the initial examination to 43 at discharge and 47 on the final examination ( $12 \%$ of recovery).

The hemorrhage pattern showed a significant correlation with complete SCI $(P=0.0001)$.

\section{Type II pattern (edema)}

Of the patients with spinal cord edema, 14 had an incomplete SCI and a favorable outcome (ASIA D or E) on final evaluation was noted. Only one patient with an edema pattern showed on admission a complete conus medullaris lesion, due to a burst fracture at T12 vertebra. Initially, an incomplete L2 SCI, ASIA B, was diagnosed at another hospital, but neurological deterioration was observed during transfer and the patient was admitted with a complete T12 SCI, ASIA A. Two years after injury, the MRI showed a T9-T12 syringomyelia. The mean MIS in this pattern increased from 71 initially to 88 at discharge and 92 at follow-up (30\% recovery).
The relation between the edema pattern and incomplete SCI was statistically significant $(P=0.001)$.

Type III pattern (contusion)

Of the 10 patients with a contusion pattern, seven were admitted with an incomplete SCI (70\%), and a satisfactory outcome was recorded (ASIA C and D in the last control). Of the three patients with a complete SCI, one eventually improved to incomplete SCI, ASIA $\mathrm{B}$ during admission. The other two patients showed no change. The MIS averaged 38 and increased to 70 at discharge and 90 at follow-up (137\% of recovery).

\section{Compression pattern}

Of the nine patients with this pattern, seven had a complete SCI, with no change in clinical outcome. A patient with a complete T5 SCI died 10 days after admission due to respiratory failure. The two patients admitted with an incomplete SCI had a favorable 
a

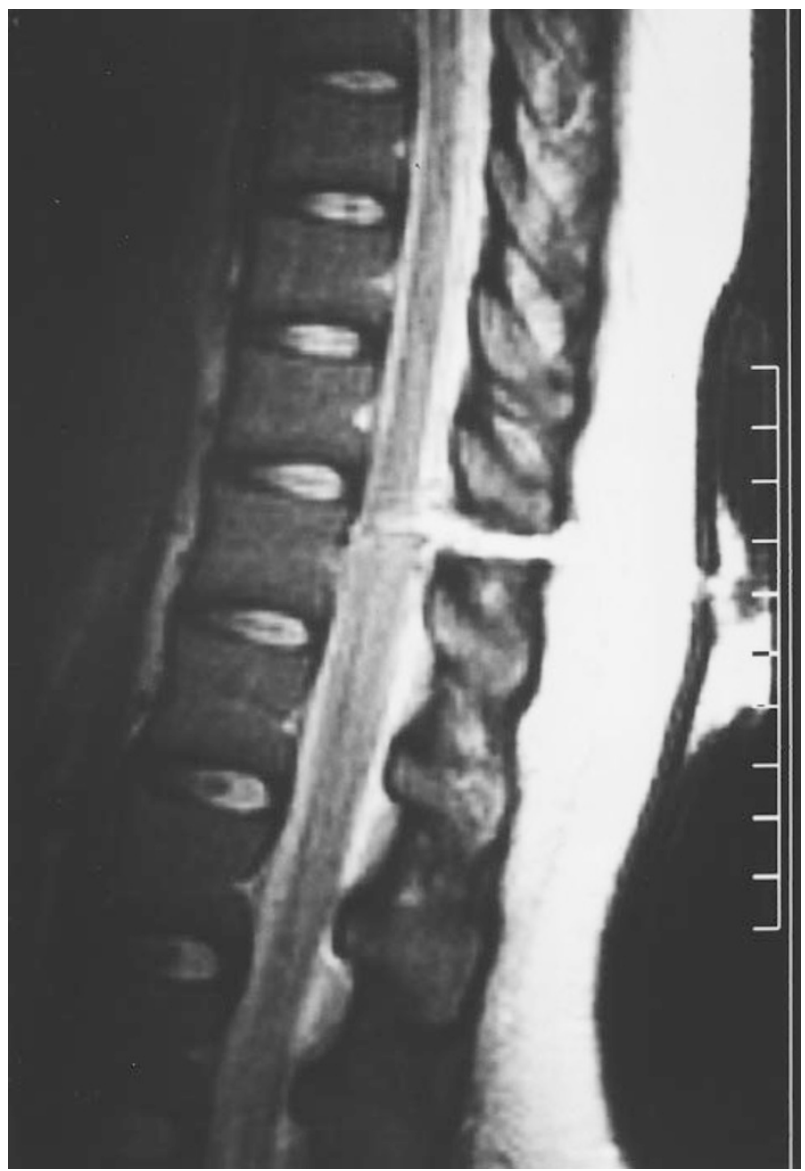

b

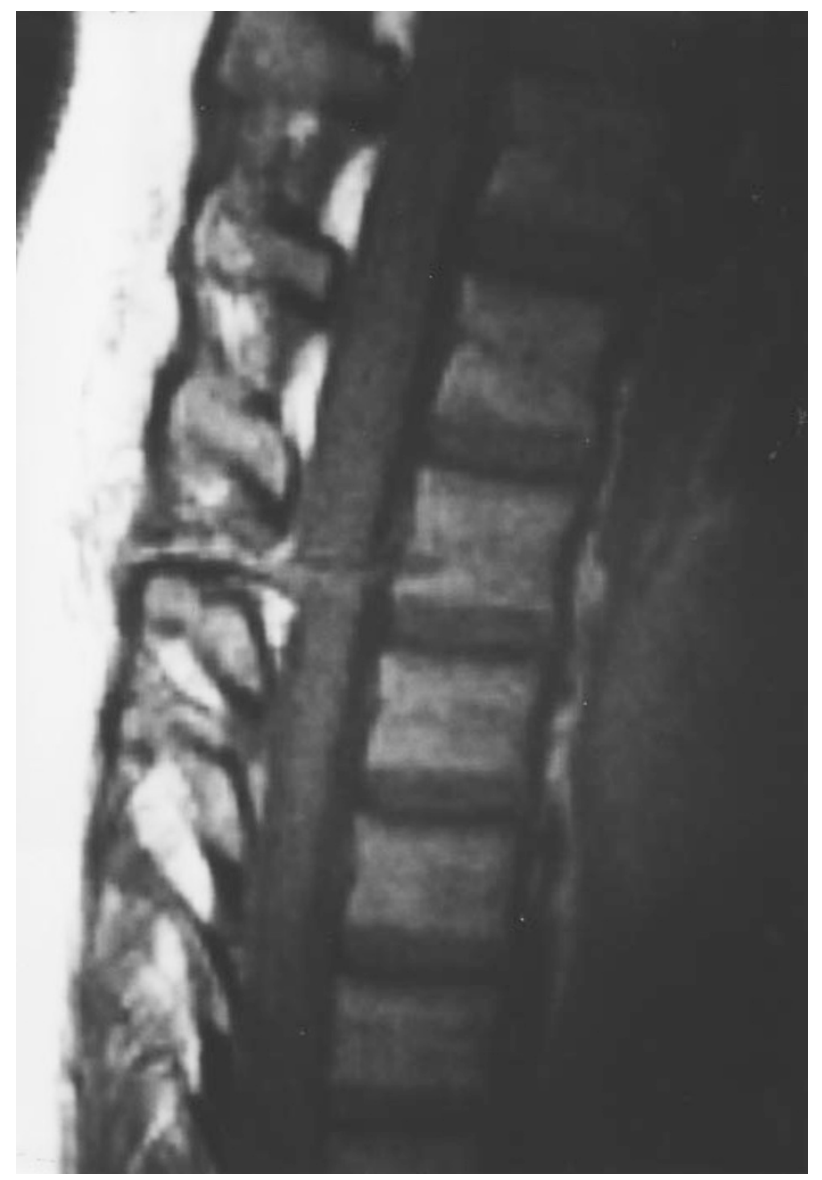

Figure 5 A 21-year-old woman stabbed in the back presented a T12 complete paraplegia. A T2 and T1-WI performed $24 \mathrm{~h}$ after the accident showed complete cord transection at the level of the T10-T11 junction, which extended from the paravertebral musculature to the vertebrae at T11 ( $\mathrm{a}$ and $\mathrm{b}$ )

outcome (ASIA D and E at the final evaluation). Their mean MIS was 52, which improved to 57 at discharge and rose to 60 at the time of follow-up $(15 \%$ of recovery).

\section{Transection pattern}

Only two patients with a complete SCI showed a transection pattern which was unchanged at the time of discharge and in final evaluation. One patient had a fracture-dislocation of the mid-thoracic region and another following a stab injury at the level of T10T11 vertebrae (Figure 5). Their mean MIS was 50 upon admission, which remained 50 in final examination ( $0 \%$ of recovery).

In addition, related clinical and imaging factors were analysed in order to establish prognostic factors in the early phase of the SCI. None of the $11 \mathrm{MRI}$ findings were significantly related to the type of SCI and final outcome. Although no statistically significant relationship was found between the type of incomplete syndrome and any given MRI pattern,
Table 1 Other spinal data seen on MRI

\begin{tabular}{lrll} 
*Cord enlargement & 12 & *Extradural hematoma & 17 \\
*Perilesional cord edema & 11 & *Disc herniation & 19 \\
*Bony fragments & 9 *Perilesional edema & 13 \\
*Posterior ligament lesion & 31 *Posterior column injury & 29 \\
*Vertebral body fracture & 20 & *Subdislocation & 31 \\
*Spondylosis & 6 & \\
\hline
\end{tabular}

Brown-Séquard syndrome tended to be associated with the edema pattern. No patient with an incomplete SCI showed a hemorrhage or transection MRI pattern (Table 4).

Methylprednisolone therapy was perfused in 21 patients $(38.2 \%)$. Neurological outcome in patients with an incomplete SCI was favorable with or without the treatment $(P=0.72)$, while in complete SCIs it remained unchanged $(P=0.4)$. Nevertheless, the series is insufficient to draw on any conclusion. Conservative fracture treatment was performed in 46 cases, while the other nine remaining patients underwent surgery (Table 


\section{INCOMPLETE SCI}

\section{COMPLETE SCI}

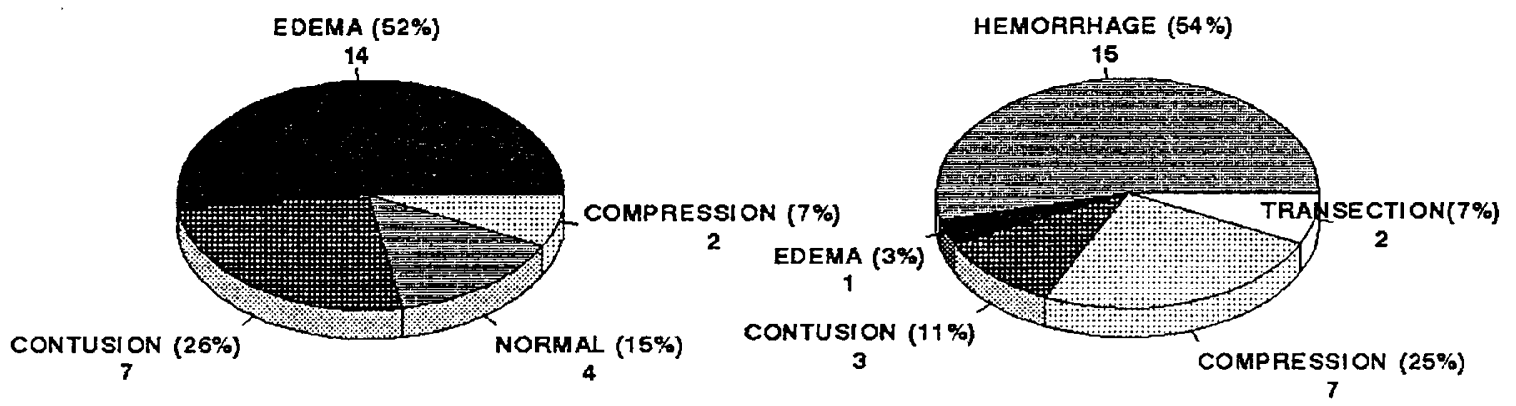

Figure 6 MRI patterns in SCI

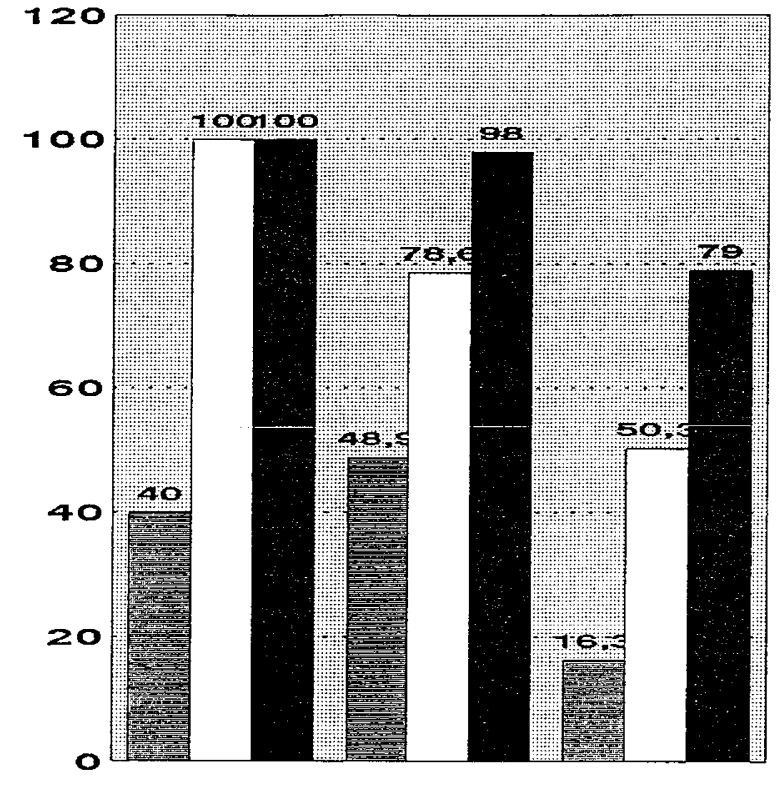

NORMAL EDEMA CONTUSION

\section{臯 ADMISSION \\ DISCHARGE \\ FOLLOW-UP}

Figure 7 Relation between motor index score in incomplete tetraparetics and MRI patterns

5). The operative treatment was performed within 7.7 days after injury (range: $5-14$ ). The fracture treatment in the two groups did not significantly affect the neurological prognosis $(P=0.09)$.

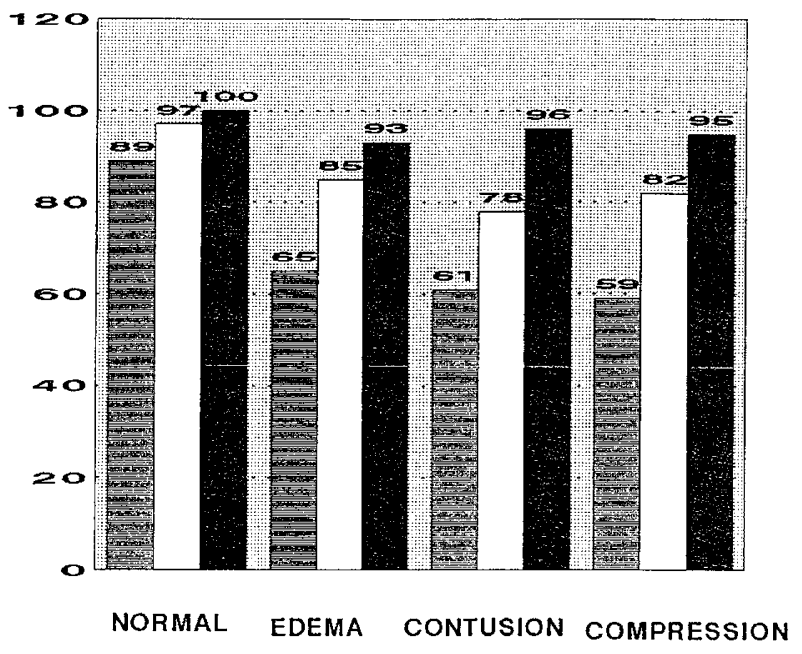

\section{图 ADMISSION \\ DISCHARGE \\ FOLLOW-UP}

Figure 8 Relation between motor index score in incomplete paraparetic and MRI patterns

The time elapsed between the acute injury and the follow-up examination was 17.4 months (range 2 days to 38 months). Two patients died upon admission. Mean time from the trauma to the MRI was 8.2 days, SD: 4.6 (range $1-15$ ).

\section{Discussion}

MR imaging is currently the only technique which allows visualization of the SCI and adjacent soft tissues, making MR examination the optimal imaging 
Table 2 Relation between SCI outcome according to the ASIA scale and MRI patterns. (Admission and discharge on the right; discharge and follow-up on the left

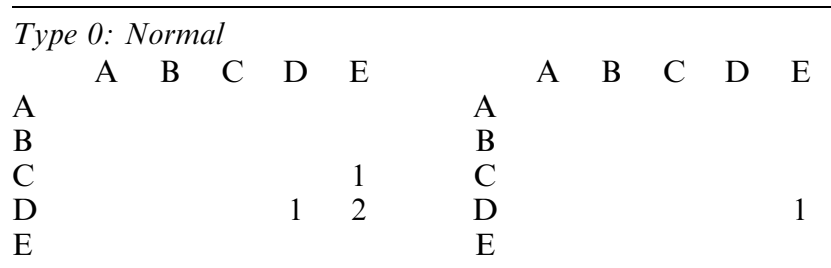

Type I: Hemorrhage

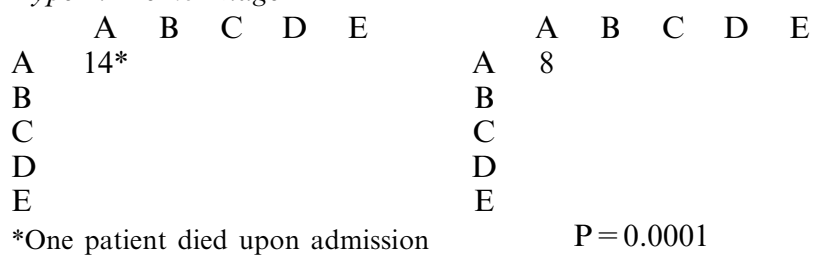

Type II: Edema

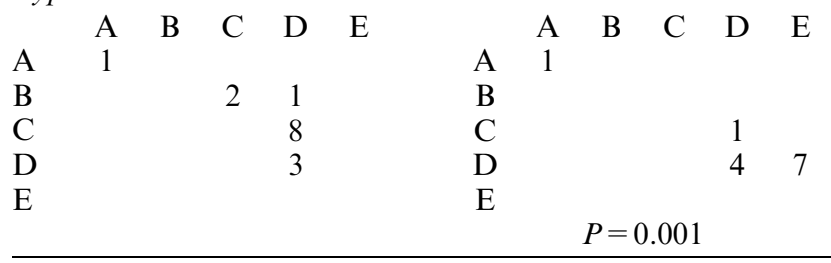

modality in the diagnosis of $\mathrm{SCI}^{3,5,8-10,14-18}$ and the neurological outcome. ${ }^{2-7}$

Research on neurological recovery requires identification of the initial motor and sensory score within each neurological level and according to the spinal cord syndrome (complete and incomplete syndromes) on serial examinations. In our study, the six MRI patterns described were related to the initial neurological examination and the recovery potential of the patient. Knowledge of functional prognosis in each SCI type is critical if optimal care is to be provided.

The patients in our series who showed a normal pattern have progressed to a complete resolution of the neurological symptoms. Nevertheless, the existence of unchanged clinical SCI without radiographic abnormalities $^{21}$ - even in MRI-should not be neglected. Thus, we feel that in patients who show a dissociation between the SCI clinical condition and a normal MRI pattern, caution is required when predicting the patient's outcome and prognosis should be based on the clinical evaluation.

The 15 patients with Type I pattern (hemorrhage) presented irreversible complete SCI. This irreversible outcome is similar to that reported by Lucas and Ducker $^{22}$ except that in our study no patient recovered infralesional motor capacity, whereas the latter authors claimed that almost $15 \%$ of their patients recovered partly such capacity. Due to its significance, complete SCI showing hemorrhage on MRI is a sign of poor prognosis.
Table 3 Relation between SCI outcome according to the ASIA scale and MRI patterns. (Admission and discharge on the right; discharge and follow-up on the left)

\begin{tabular}{lccccccccccc}
\hline \multicolumn{1}{c}{ Type III: Contusion } & & & & & & & \\
& A & B & C & D & E & & A & B & C & D & E \\
A & 2 & 1 & & & & A & & & & & \\
B & & 1 & 1 & 1 & & B & & & 1 & & \\
C & & & & 3 & & C & & & & 3 & \\
D & & & & 1 & & D & & & & & \\
E & & & & & E & & & & &
\end{tabular}

Type IV: Compression

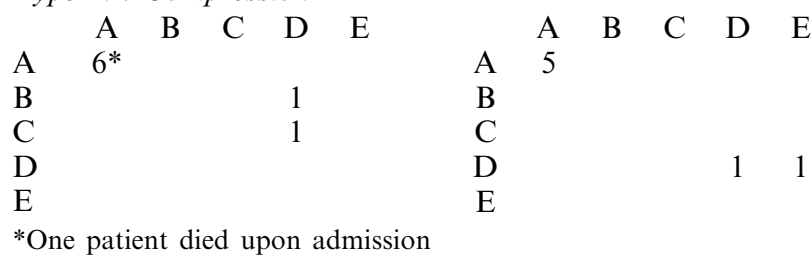

Type V: Transection

$\begin{array}{llllll} & \text { A } & \text { B } & \text { C } & \text { D } & \text { E } \\ \text { A } & 2 & & & & \\ \text { B } & & & & & \\ \text { C } & & & & \\ \text { D } & & & & \\ \text { E } & & & & \\ * & \text { Unchanged throughout evolution }\end{array}$

Table 4 Patients with incomplete SCI and MRI patterns

\begin{tabular}{ll}
\hline SCI syndrome & MRI pattern \\
\hline *Brown-Séquard & 5 Edema \\
*Anterior & 1 Contusion \\
& 3 Edema \\
& 2 Contusion \\
& 2 Compression \\
$*$ Central & 2 Edema \\
& 1 Contusion \\
$*$ Conus medularis & 3 Edema \\
& 2 Normal \\
$*$ Non classifiable' & 2 Contusion \\
& 2 Normal \\
& 1 Edema \\
& 1 Contusion \\
\hline
\end{tabular}

A significant relationship between Type II pattern (edema) and incomplete SCI, with favorable outcome to functional degrees, is noted. Only one patient with an edema pattern suffered a complete SCI. Two years after injury, the MRI showed a T9-T12 syringomyelia, which may justify it.

Seventy per cent of the patients with Type III pattern (contusion) were admitted with an incomplete SCI, with satisfactory outcome despite ASIA E grade in the follow-up was not reached. Moreover, one of 
Table 5 Patients with vertebromedullary trauma, surgically treated

\begin{tabular}{|c|c|c|c|c|c|}
\hline Fracture type and site & Level & $A S I A a-d$ & $M R I$ & Operative approach & $T(d)$ \\
\hline -Fr-disloc C4-5 & $\mathrm{C} 5$ & $\mathrm{D}-\mathrm{D}$ & II: EDEMA & $\mathrm{ANT}$ & 8 \\
\hline -Fr-disloc T10-11 & $\mathrm{T} 10$ & A-A & I: $\mathrm{HMG}$ & POST & 5 \\
\hline $\begin{array}{l}\text {-Fr-disloc T10-11 } \\
\text { + burst T11 }\end{array}$ & $\mathrm{T} 10$ & A-A & IV: COMPR & POST & 5 \\
\hline -Fr burst T12 & L3 & $\mathrm{B}-\mathrm{B}$ & III: CONT & POST & 9 \\
\hline -Fr burst $\mathrm{T} 12$ & $\mathrm{~T} 12$ & A-A & II: EDEMA & ANT + POST & 8 \\
\hline -Fr burst $\mathrm{T} 12$ & T12 & B-D & II: EDEMA & POST & 5 \\
\hline -Fr-disloc T12-L1 & T11 & A-A & I: $\mathrm{HMG}$ & POST & 14 \\
\hline -Fr burst L1 & $\mathrm{T} 12$ & A-A & III: CONT & $\mathrm{ANT}+\mathrm{POST}$ & 6 \\
\hline -Fr burst L1 & L1 & D-D & III: CONT & $\mathrm{ANT}+\mathrm{POST}$ & 10 \\
\hline
\end{tabular}

*ASIA a-d: American Spinal Injury Association scale on admission and discharge; MRI: Magnetic Resonance Image; T (d): Timing to surgery in days; FR-disloc: fracture-dislocation; CONT: contusion; HMG: hemorrhage; COMPR: compression; ANT: anterior; POST: posterior

the two patients with a complete SCI upon admission evolved to incomplete ASIA B. Therefore, we feel that this pattern is related to an incomplete SCI, with some type of neurological impairment still evident at the final evaluation.

According to Schaefer ${ }^{4}$, quantification of the SCI on MRI would be of value in all patients in order to establish prognosis. Another recent study demonstrates that MRI findings of cord hematoma or contusion involving more than one spinal segment were associated with severe neurological damage. ${ }^{15}$ Sequential MRI is recommended in the acute and subacute SCI phases to determine the prognosis-as patterns can change over the time $9,18,19-$ and to monitor pharmacological investigation with imaging in subsequent examinations.

In our series, it was not possible to correlate statistically compression pattern to any type of SCI, although a trend to association with complete SCI and a poor functional prognosis was noted. This pattern was only observed at the thoracic spine. Furthermore, a compression pattern may hinder visualization of a hemorrhage, which may explain why it is associated with complete SCI. To clarify this hypothesis, it would be appropriate to perform MRI several times and to correlate it, or histopathological studies in patients who have died.

The transection pattern was observed in two patients with complete SCI. This accounts for $7 \%$ of all MRI patterns in patients with complete SCI, but more cases would be needed to draw any conclusion.

Although we have studied the prognosis of all MRI patterns described according to Kulkarni's classification, ${ }^{7}$ we propose a clinical and imaging classification based on it and with SCI's severity: Type 0 (normal), Type I (edema), Type II (contusion), Type III (compression), Type IV (hemorrhage) and Type $V$ (transection).

As far as the neurological outcome of our patients is concerned, the use of methylprednisolone therapy did not prove clearly effective since incomplete SCI improved in both groups. Nevertheless, the small sample does not allow us to reach statistical conclusions. The same conclusions were obtained regarding the treatment of vertebral fractures. In our study, all patients but two have unchanged complete SCI. These two patients with complete SCI ASIA A who progressed to incomplete SCI, had only recovered superficial sensory, ASIA B.

\section{Conclusions}

Clinical SCI complemented with MRI are predictive for early functional prognosis in the following patterns: In the cases of complete SCI, cord hemorrhage or transection MRI patterns back up the clinical diagnosis and establish an early prognosis of irreversible neurological damage. In the cases of incomplete SCI, when the edema pattern is seen, a functional prognosis of neurological recovery (ASIA D or E) may be established. The Brown-Séquard syndrome tends to be associated with the edema pattern. Spinal contusion imaging tends to be associated with incomplete SCI, unlike the compression pattern, in which results varied widely, with prognosis depending on the initial clinical injury.

\section{References}

1 Frankel HL et al. The value of postural reduction in the initial management of closed injuries of the spine with paraplegia and tetraplegia. Part I. Paraplegia 1969; 7: 179-192.

2 Hayashi K et al. MRI findings in patients with a cervical spinal cord injury who do not show radiographic evidence of a fracture or dislocation. Paraplegia 1995; 33: 212-215.

3 Kulkarni MV, Bondurant FJ, Rose SL, Narayana PA. 1.5 tesla magnetic resonance imaging of acute spinal trauma. Radiographics 1987; 8: $1059-1082$.

4 Schaefer DM, Flanders AE, Osterholm JL, Northrup BE. Prognostic significance of magnetic resonance imaging in the acute phase of cervical spine injury. $J$ Neurosurg 1992; 76: 218 223.

5 Bondurant FJ et al. Acute spinal cord injury. A study using physical examination and magnetic resonance imaging. Spine 1990; 15: $161-167$. 
6 Marciello MA et al. Magnetic resonance imaging related to neurologic outcome in cervical spinal cord injury. Arch Phys Med Rehabil 1993; 74: $940-946$.

7 Kulkarni MV et al. Acute spinal cord injury: MR imaging at 1.5 T. Neuroradiology 1988; 164: $837-843$.

8 Kalfas I, Wilberger J, Goldber A, Prostko ER. Magnetic resonance imaging in acute spinal cord trauma. Neurosurgery 1988; 23: $295-299$

9 Sett P, Crockard HA. The value of magnetic resonance imaging in the follow-up management of spinal injury. Paraplegia 1991; 29: $396-410$.

10 Silberstein M, Hennessy O. Implications of focal spinal cord lesions following trauma: evaluation with magnetic resonance imaging. Paraplegia 1993; 31: 160-167.

11 American Spinal Injury Association, International Medical Society of Paraplegia (ASIA/IMSOP). International standards for neurological and functional classification of spinal cord injury. Revised 1992. American Spinal Injury Association, Chicago III

12 Ditunno JF, Young W, Donovan WH, Creasey G. The international standards booklet for neurological and functional classification of spinal cord injury. Paraplegia 1994; 32: 70-80.

13 Bracken MB et al. A randomized, controlled trial of methylprednisolone or naloxone in the treatment of acute spinal-cord injury. Results of the second National Acute Spinal Injury Study. $N$ Engl J Med 1990; 322: 1405-1411.

14 Heier LA, Johnson CE. Efficacy of magnetic resonance imaging in the diagnosis of spinal cord trauma. In: Lee BY, Ostrander LE, Cochran GB, Shaw WW. The spinal cord injured patient. Comprehensive management. WB Saunders, 1991: pp 90-114.
15 Herzog RJ. MRI of the spine. In: Frymoyer JW, (ed.) The Adult Spine. Principles and practice. Vol 1. New York: Raven Press, Ltd., 1991: pp 484-486.

16 Perovitch $\mathrm{M}$, Wang $\mathrm{H}$, Perl S. The evolution of neuroimaging of spinal cord injury patients over the last decade. Paraplegia 1992; 30: $39-42$.

17 Schaefer DM et al. Magnetic resonance imaging of acute cervical spine trauma. Correlation with severity of neurologic injury. Spine 1989; 14: 1090-1095.

18 Taneichi H, Abumi K, Kaneda K, Terae S. Monitoring the evolution of intramedullary lesions in cervical spinal cord injury. Qualitative and quantitative analysis with sequential MR imaging. Paraplegia 1994; 32: 9- 18

19 Montanera W, Marmor E. Imaging of spinal cord injury. In: Benzel EC, Tator CH (eds). Contemporary management of spinal cord injury. Neurosurgical topics. American Association of Neurological Surgeons Publications Committee. USA, 1995: pp $63-78$.

20 Sato $\mathrm{T}$ et al. Prognosis of cervical spinal cord injury in correlation with magnetic resonance imaging. Paraplegia 1994; 32: $81-85$.

21 Pang D, Wilberger JE Jr. Spinal cord injury without radiographic abnormalities in children. J Neurosurg 1982; 57: 114-129.

22 Lucas JT, Ducker TB. Motor classification of spinal cord injuries with mobility, morbidity and recovery indices. Am Surg 1979; 3: $151-158$. 